concentration was increased into the range of $0.05-0.1 \mathrm{M}$, instead of the range of $0.002-0.1 \mathrm{M}$, polyaniline rod-like structures formed together with the nanofibers. Typical nanorod diameters were 250-500 $\mathrm{nm}$ and lengths were 5-10 $\mu \mathrm{m}$. Although the mechanism of polyaniline nanofiber formation is unknown, the researchers found that nanoparticles resembling hollow spheres formed during irradiation, and their aggregation seemed to play an important role in the fiber formation. According to the researchers, further studies on the characteristics and precise formation conditions of these hollow nanoparticles are currently being conducted.

LuCY Yue Hu

\section{Room-Temperature Nanoimprinting Enables \\ Fabrication of 1D Photonic-Crystal Oligomer-Based Lasers}

The photoluminescence and stimulatedemission properties of some conjugated compounds make them well suited for use as distributed feedback resonators (DFBs) in solid-state organic lasers. Organic lasers are attractive alternatives to conventional semiconductor lasers, as they are likely to be more easily and cheaply manufactured. However, many fabrication techniques for forming grooved DFB structures can cause degradation of the optical properties by incorporating oxygen at high temperatures or by poor transfer of the topography. To avoid these issues, D. Pisignano (Università di Lecce, Italy), G. Barbarella (Istituto per la Sintesi Organica e la Fotoreattività, Bologna, Italy), and colleagues used a room-temperature nanoimprinting process to print one-dimensional photonic crystals made of a thiophene-based pentamer (T5oCx). By using these photonic crystals as resonators in DFB lasers, the research team printed single-mode lasers with low lasing thresholds.

Resonator fabrication began by spincasting the $\mathrm{T} 5 \mathrm{oCx}$ oligomer onto quartz substrates. A silicon grating formed by electron-beam lithography and reactive ion etching was pressed into the organic film, leaving behind a replica of the grating structure in the oligomer. The gratings had a period of $400 \mathrm{~nm}$ and areas as large as $3 \mathrm{~mm} \times 7 \mathrm{~mm}$.

Pisignano, Barbarella, and their colleagues then studied the emission proper- ties of these resonators, as reported in the February 1 issue of Optics Letters (p. 260). After illuminating a small area with a HeCd laser $(\lambda=325 \mathrm{~nm})$, the researchers collected the angle-resolved photoluminescence (PL) spectra of the oligomer gratings. They found that there is a strong normalincidence PL peak at $611 \mathrm{~nm}$ that is not present in as-cast films. As the collection angle increased, the peak split into two, with the peak separation eventually spanning the amplified stimulated emission band of the $\mathrm{T} 5 \mathrm{oCx}$. The researchers also demonstrated the performance of the oligomer gratings used as a DFB laser by pumping a large area $(7 \mathrm{~mm} \times 0.5 \mathrm{~mm})$ with a frequency-tripled Nd:YAG laser ( $\lambda=355 \mathrm{~nm}, 3$ ns pulses) under vacuum. These organic lasers operated at a wavelength of $637 \mathrm{~nm}$ in the second order of the diffraction grating and had a lasing threshold as low as $37 \mu \mathrm{J} / \mathrm{cm}^{2}$.

The low lasing threshold of these devices represents a marked improvement over previously observed thresholds for third-order DFB lasers and planar slabs made out of the same material. The simplicity of the one-step, dry imprinting process for fabricating complete lasers,

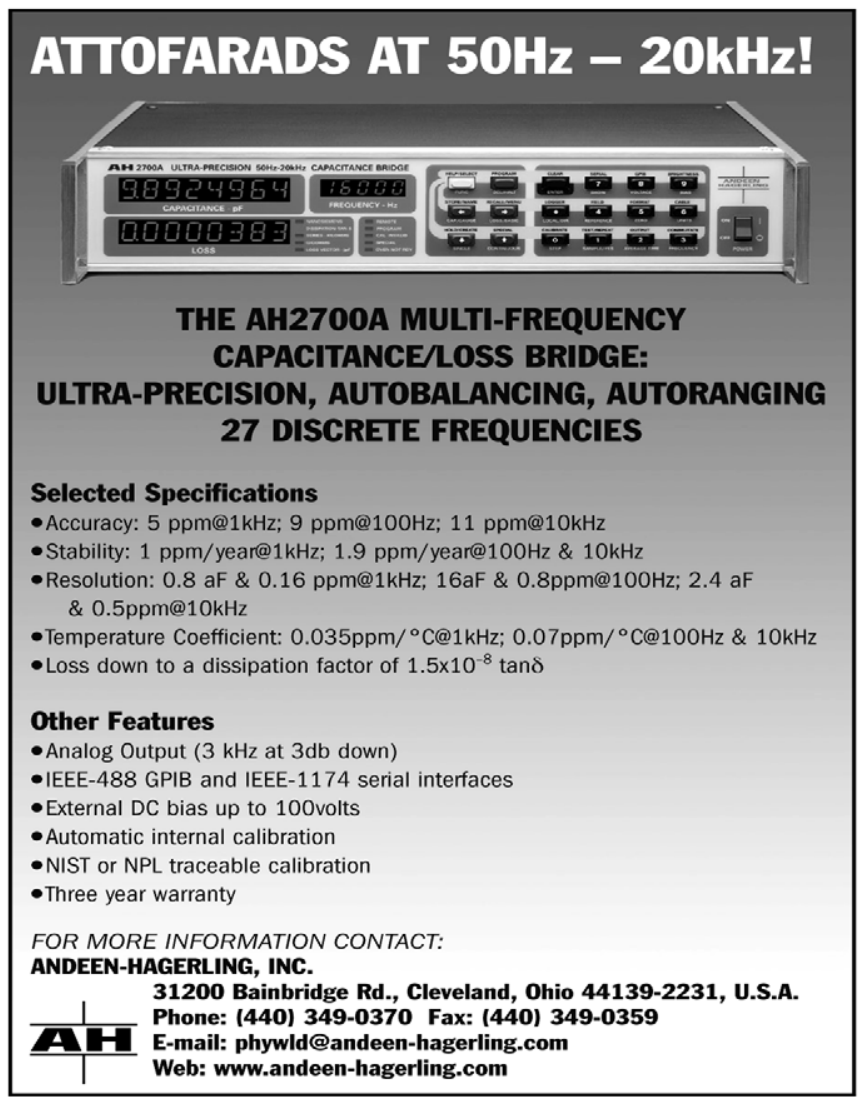

For more information, see http://advertisers.mrs.org

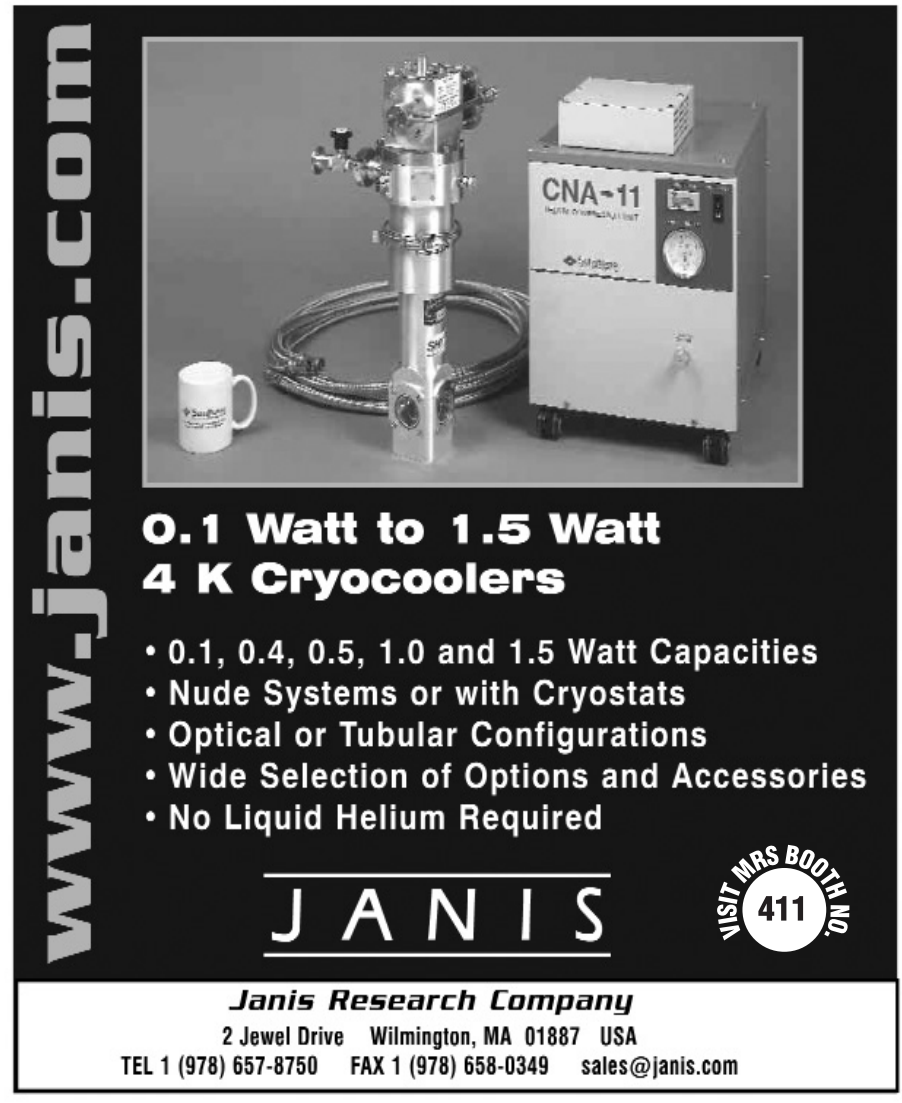

For more information, see http://advertisers.mrs.org 
along with the demonstration of good performance of the devices, opens the way to further exploration of these methods for organic laser production.

AMANDA GIERMANN

\section{Poly(3,3" -dialkyl-terthiophene) OTFTs Stable under Ambient Atmospheric Conditions}

Organic thin-film transistors (OTFTs) have the advantages of low-cost solution fabrication and excellent flexibility, which enable their application in devices such as flexible displays and electronic paper. However, most solution-processed organic semiconductor materials used in OTFTs either have poor structural order from solution or their performance is degraded by atmospheric oxygen doping. Recently, a group of scientists from Xerox Research Center of Canada demonstrated that poly $\left(3,3^{\prime \prime}\right.$-dialkyl-terthiophene)s have sufficient air stability to enable solution fabrication of functionally useful OTFTs under ambient conditions.

As reported in the January 25 issue of Chemistry of Materials (p. 221; DOI: $10.1021 / \mathrm{cm} 048678 \mathrm{r})$, B.S. Ong and co- workers synthesized poly $\left(3,3^{\prime \prime}-\mathrm{di}-n-\right.$ octylterthiophene) by a controlled $\mathrm{FeCl}_{3-}$ mediated polymerization in chlorobenzene. While most solution-processed semiconductors used in OTFTs need high-temperature in situ reactions or postdeposition thermal annealing, the material the research team designed, poly $\left(3,3^{\prime \prime}\right.$-di- $n$-octylterthiophene), could be processed at room temperature and showed higher field-effect transistor (FET) mobility without thermal treatment. Through the study of poly $\left(3,3^{\prime \prime}\right.$-di$n$-octylterthiophene), the researchers demonstrated the profound influence of side-chain regiochemistry on molecular ordering behavior and the subsequent impact on field-effect characteristics.

Using poly( $3,3^{\prime \prime}$-di- $n$-octylterthiophene) as the channel material, the researchers then fabricated and characterized their OTFT devices at room temperature and in open air. These 20-50-nm-thick devices (90 $\mu \mathrm{m}$ or $190 \mu \mathrm{m}$ channel length, channel width of $1 \mathrm{~mm}$ or $5 \mathrm{~mm}$ ), fabricated on a 100-nm-thick thermal oxide on an $n$-doped silicon wafer, showed near-ideal FET behavior and greater ambient stability compared with other organic semiconductors. The researchers said that the excellent self-organization ability in thin films from solution at room temperature without thermal assistance and its stability in air may render poly $\left(3,3^{\prime \prime}\right.$-dialkyl-terthiophene) an ideal channel semiconductor for lowcost OTFTs. This technology could ultimately lead to inexpensive large-area devices like flat-panel and flexible displays and low-end microelectronics such as rf identification tags.

TAO XU

\section{Low-Density Sublimed $p$-tert- butylcalix[4]arene Demonstrates Ability for Methane Storage at Room Temperature}

Calixarenes are complex organic compounds formed by phenolic molecules arranged in open structures capable of encapsulating different ions or molecules. One form of these compounds is calix[4]arene, which can stably entrap vapor or gas molecules, releasing them only after heat decomposes its structure. As p-tert-butylcalix[4]arene, it can selectively adsorb $\mathrm{CO}_{2}$ from a mixture with $\mathrm{H}_{2}$.

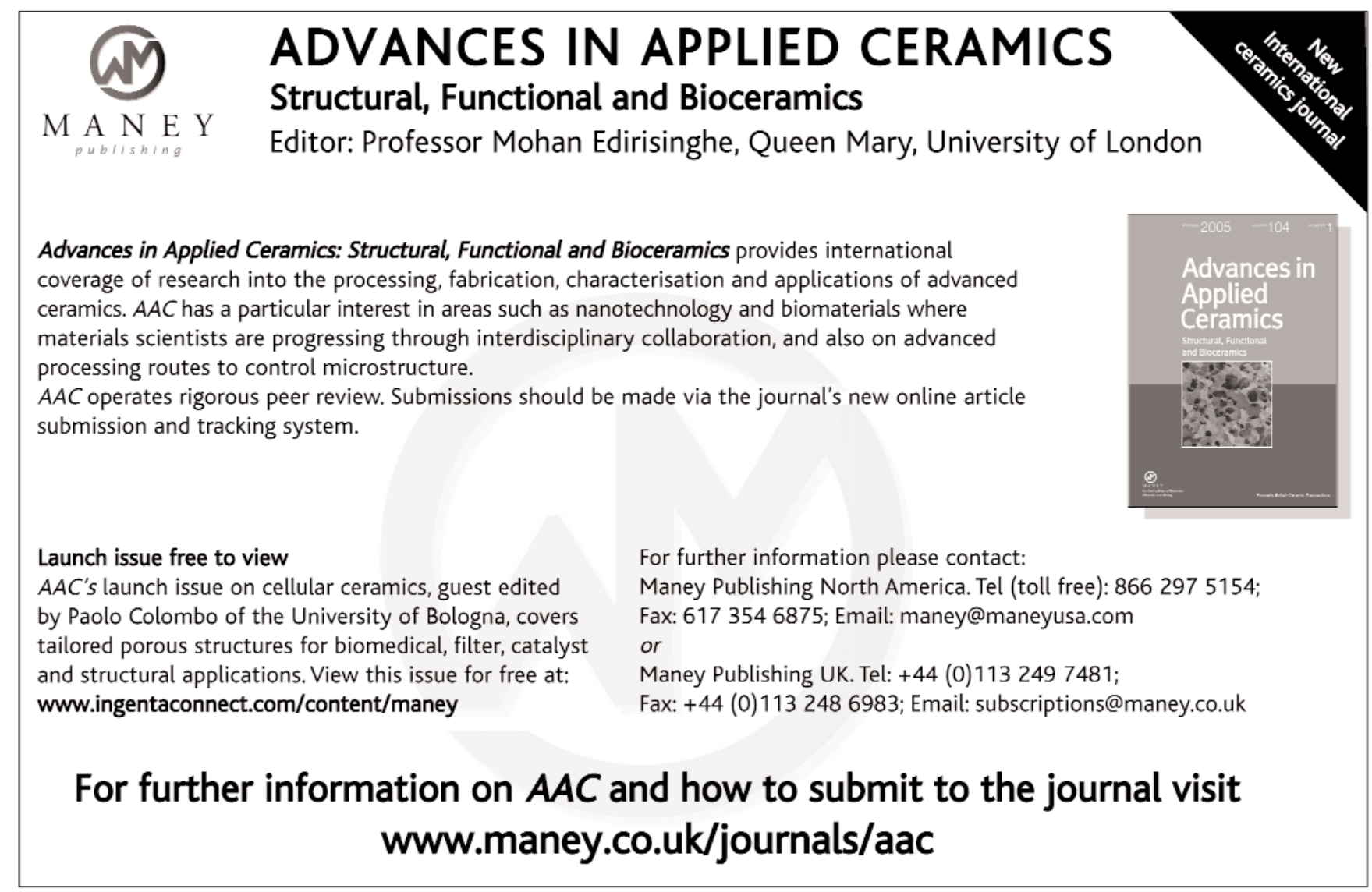

For more information, see http://advertisers.mrs.org 